\title{
Étude des Trématodes parasites de Littorina saxatilis (Olivi) et de leurs effets sur cet hôte
}

\author{
par Catherine COMBESCOT-LANG (*) \\ Laboratoire de Zoologie-Parasitologie, U.E.R. des Sciences pharmaceutiques, \\ 2 bis, Boulevard Tonnellé, F 37000 Tours.
}

\section{Résumée.}

L'étude mensuelle de populations de Littorina saxatilis (mollusque, gastéropode) très fortement parasitées a permis d'établir quelques relations entre ce mollusque et certains trématodes parasites qu'il héberge.

Les animaux ont été prélevés dans deux stations de la région de Roscoff (Finistère). Au total plus de 1.000 individus ont été examinés pour chaque station.

Onze espèces de cercaires ont été reconnues. Il est apparu que Microphallus similis était la plus représentée : 79,86\% des espèces trouvées. Ce taux de parasitisme varie peu au cours de l'année. Les populations fortement parasitées par Microphallus similis présentent une castration importante. Une double association de cercaires reste rare, mais cependant possible.

Il apparaît que les individus âgés sont les plus parasités. Il y aurait par ailleurs une croissance des parasites plus importante chez les femelles que chez les mâles.

\section{Summary.} molluse.

A study of parasitic Trematods of Littorina saxatilis and their impact on this

A monthly study of populations of Littorina saxatilis (mollusc, gasteropod) very strongly parasited has allowed to establish relations between the said mollusc and some parasite trematods which live in it.

A selection of animals was done in two stations of the Roscoff region (Finistere). A total fo more than a 1.000 individuals have been examined in each station.

Eleven species of cercairs have been spotted. It has appeared that the Microphallus similis was the most represented: $79,86 \%$ of the total. This rate of parasitism does not

${ }^{*}$ ) Je tiens à remercier M. le $\mathrm{P}^{r} \mathrm{~J}$. Bergerard, Directeur de la Station Biologique de Roscoff, qui a dirigé ce travail, ainsi que M. le $\operatorname{Pr}$ A.-G. Chabaud et $\mathbf{M}^{\mathrm{me}}$ J. Richard qui m'ont initiée dे la reconnaissance des cercaires. 
change much throughout the year. The populations of molluscs strongly parasited by Microphallus similis show an important castration. A double association of cercairs remains rare but is yet possible.

It appears that aged individuals are the most parasited. It seems that there would be a growth of parasites more important in females than in males.

On sait que les formes larvaires de trématodes entraînent habituellement la castration parasitaire des mollusques hôtes. Chez Littorina saxatilis plusieurs auteurs ont étudié les effets du parasitisme par différents trématodes. On peut citer en particulier James (1969) dont les travaux ont été effectués sur la côte anglaise à Cardigan Bay.

Nous avons choisi pour notre étude, dans la région de Roscoff, deux populations locales à fort taux de parasitisme, l'une sur le rocher du «Bourguignon » près de la côte en face du Laboratoire, l'autre à plusieurs milles en mer sur l'île du Beclem à l'entrée de la baie de Morlaix. Des prélèvements mensuels ont été effectués pour les deux populations et au total plus de 1000 individus ont été examinés pour chaque station. Notre travail a été effectué à la Station Biologique de Roscoff.

\section{Recherche des parasites}

Les parasites sont situés dans l'hépatopancréas du mollusque. Afin de les mettre en évidence, nous avons brisé la coquille de ce dernier. Puis nous avons placé le corps de l'animal dans une boîte de Pétri contenant de l'eau de mer artificielle (**). Parfois, à l'œil nu, certaines anomalies de l'hépatopancréas, marbrure, aspect plus ou moins déchiqueté laissent supposer la présence de parasites (Willey et Gross, 1957). Souvent on peut apercevoir les sporocystes et les rédies qui parsèment le fond de la boîte de Pétri, ainsi que les cercaires qui nagent ou rampent sur le fond.

Parfois la dissection du Mollusque à la loupe binoculaire est nécessaire pour mettre en évidence les parasites.

Les métacercaires sont parfois difficiles à déceler, celles-ci se situent généralement au niveau du rein, du cœur, de l'estomac ou de l'hépatopancréas.

Les larves sont prélevées avec une micropipette.

Nous avons pu ainsi isoler 11 cercaires différentes, désignées au départ par les lettres de l'alphabet, 9 ont été identifiées, tandis que 2 rencontrées une seule fois n'ont pas été reconnues.

IDENTITÉ DES PARASITES :

1. Cercaire A : Microphallus similis Jaegerskioeld, 1900.

Cercaria ubiquita Lebour, 1907.

Cercaria ubiquitoides Stunkard, 1932.

Cercaria sp. Rees, 1936 a.

$t^{\text {t***)}}$ Eau de mer en poudre (Leveaux, Quai du Louvre). 
2. Cercaire B : Renicola roscovita: Cercaria roscovita Stunkard, 1932, Bayssade et Lang, 1973.

3. Cercaire C : Cercaria lebouri Stunkard, 1932.

4. Cercaire D : Parapronocephalum symmetricum Chabrik, 1954.

5. Cercaire E : non identifiée.

6. Cercaire F : nous pensons qu'il s'agit de Cercaria littorinae saxatilis III de James.

7. Cercaire G : Cryptocotyle lingua.

8. Cercaire I : non identifiée.

9. Cercaire $\mathbf{J}$ : Cercaria littorinae saxatillis II.

10. Cercaire UV: Podocotyle atomon.

11. Cercaire S : Microphallus pygmeus Levinsen, 1881.

Nous avons d'autre part désigné par:

- $\mathrm{K}$ : les sporocystes immatures dont les cercaires ne sont pas encore identifiables.

$-\mathrm{H}$ : les métacercaires.

REMARQUe : Les cercaires A et B ayant été les plus fréquemment rencontrées chez Littorina saxatilis, on peut supposer qu'elles sont responsables des modifications observées dans les populations étudiées.

\section{Taux de parasitisme global en fonction du sexe}

Comme nous l'avons déjà noté le parasitisme entraîne le plus souvent une régression plus ou moins complète des appareils génitaux (mâles ou femelles). Un certain nombre d'individus apparaissent ainsi au premier abord de sexe difficilement identifiable. Si l'on considère cependant que nos récoltes n'ont englobé que les individus de plus de $5 \mathrm{~mm}$ de hauteur de coquille, taille à laquelle les mâles voient se réaliser une petite ébauche triangulaire de pénis (Bergerard, 1975) et que la régression de l'appareil génital des mâles parasités laisse toujours au moins subsister cette ébauche de pénis, il est possible par différence d'attribuer les animaux qui ne présentent pas, au moins cette ébauche, au sexe femelle.

On obtient ainsi les résultats rapportés au Tableau $I$.

\begin{tabular}{|c|c|c|c|}
\hline & $\begin{array}{c}\text { Nombre d'animaux } \\
\text { étudiés }\end{array}$ & $\begin{array}{c}\text { Nombre d'animaux } \\
\text { parasités }\end{array}$ & $\%$ \\
\hline$\delta$ ot.......... & 896 & 402 & $44,65 \%$ \\
\hline$q \ldots \ldots \ldots \ldots$ & 864 & 435 & $50,3 \%$ \\
\hline Total ....... & 1760 & 837 & $47,5 \%$ \\
\hline
\end{tabular}

Malgré la légère différence entre le taux de parasitisme des mâles et des femelles on ne peut conclure à une influence du sexe sur le parasitisme. 
Taux de parasitisme relatif à chaque espèce de trématode

Les taux de parasitisme varient considérablement entre les différentes espèces de Trématodes (voir Tableau II).

On peut ainsi noter que seules les deux espèces $\mathrm{A}$ et $\mathrm{B}$ atteignent dans les deux populations étudiées un taux important dans les deux sexes et qu'en fait la première espèce est responsable de plus des $4 / 5^{\circ}$ des cas de parasitisme.

TABlEAU II. - Taux de parasitisme en fonction de chaque espèce de trématode : $\mathrm{P}=$ nombre d'hôtes parasités ; $\mathrm{S}=$ nombre d'hôtes sains.

\begin{tabular}{|c|c|c|c|c|c|c|c|c|c|}
\hline & & bre de & & $\%$ & $\frac{P}{P_{+}+S}$ & & $\begin{array}{l}\% p \\
\text { anir }\end{array}$ & $\begin{array}{l}\text { ar rappor } \\
\text { laux par: }\end{array}$ & $\begin{array}{l}\text { taux } \\
\text { sités }\end{array}$ \\
\hline & $\pi$ & & $T$ & & & total & & & total \\
\hline & & & & & & 1760 & & 435 & 837 \\
\hline $\mathrm{A} \ldots \ldots \ldots \ldots$ & 277 & 366 & 643 & $30,9 \%$ & $42,4 \%$ & $36,5 \%$ & $68,9 \%$ & $84,1 \%$ & $76,8 \%$ \\
\hline B $\ldots \ldots \ldots \ldots$ & 18 & 30 & 48 & $2,0 \%$ & $3,5 \%$ & $2,7 \%$ & $4,5 \%$ & $6,9 \%$ & $5,7 \%$ \\
\hline $\mathrm{c} \ldots \ldots \ldots \ldots$ & 5 & - & 5 & $0,6 \%$ & - & $0,3 \%$ & $1,2 \%$ & - & $0,6 \%$ \\
\hline $\mathrm{D} \ldots \ldots \ldots \ldots$ & 5 & 5 & 10 & $0,6 \%$ & $0,6 \%$ & $0,6 \%$ & $1,2 \%$ & $1,1 \%$ & $1,2 \%$ \\
\hline $\mathrm{E} \ldots \ldots \ldots \ldots$ & 4 & 3 & 7 & $0,4 \%$ & $0,3 \%$ & $0,4 \%$ & $1,0 \%$ & $0,7 \%$ & $0,8 \%$ \\
\hline $\mathrm{F}, \ldots \ldots \ldots \ldots$ & 1 & - & 1 & $0,1 \%$ & - & $0,06 \%$ & $0,2 \%$ & - & $0,1 \%$ \\
\hline $\mathrm{G} \ldots \ldots \ldots \ldots$ & 2 & 1 & 3 & $0,2 \%$ & $0,1 \%$ & $0,2 \%$ & $0,5 \%$ & $0,2 \%$ & $0,4 \%$ \\
\hline $\mathrm{H}, \ldots \ldots \ldots \ldots$ & 12 & 12 & 24 & $1,3 \%$ & $1,4 \%$ & $1,4 \%$ & $3,0 \%$ & $2,8 \%$ & $2,9 \%$ \\
\hline $\mathrm{I} \ldots \ldots \ldots \ldots \ldots$ & - & 1 & 1 & - & $0,1 \%$ & $0,06 \%$ & - & $0,2 \%$ & $0,1 \%$ \\
\hline $\mathrm{J} \quad \ldots \ldots \ldots \ldots$ & - & 1 & 1 & - & $0,1 \%$ & $0,06 \%$ & - & $0,2 \%$ & $0,1 \%$ \\
\hline $\mathrm{K} \ldots$ & 78 & 12 & 90 & $8,7 \%$ & $1,4 \%$ & $5,1 \%$ & $19,4 \%$ & $2,8 \%$ & $10,8 \%$ \\
\hline $\mathrm{T} \ldots \ldots \ldots \ldots$ & - & 2 & 2 & - & $0,2 \%$ & $0,1 \%$ & - & $0,5 \%$ & $0,2 \%$ \\
\hline UV $\ldots \ldots \ldots \ldots$ & - & 1 & 1 & & $0,1 \%$ & $0,06 \%$ & - & $0,2 \%$ & $0,1 \%$ \\
\hline s $\ldots \ldots \ldots \ldots$ & - & 1 & 1 & & $0,1 \%$ & $0,06 \%$ & 一 & $0,2 \%$ & $0,1 \%$ \\
\hline Total $\ldots . . . .$. & 402 & 435 & 1760 & & & & & & \\
\hline
\end{tabular}


Ce tableau permet également de mettre en évidence un fait intéressant. Les parasites immatures inventoriés \& $K$ » qui ne représentent qu'un pourcentage de près de $3 \%$ des cas chez les femelles atteignent un pourcentage de près de $20 \%$ chez les mâles.

Vu la prédominance très accentuée de Microphallus similis parmi l'ensemble des parasites, on peut raisonnablement supposer que ces sporocystes immatures appartiennent essentiellement à cette espèce qui serait donc d'une croissance beaucoup plus lente chez les mâles.

Si l'on admet cette interprétation, la part du parasitisme due à Microphallus se situe aux environs de $87 \%$ du parasitisme total.

\section{Possibilité d'hébergement simultané de plusieurs parasites d'espèces diffé- rentes.}

Nous avons pu par ailleurs rechercher s'il existait la présence simultanée dans 1 même hôte de plusieurs parasites.

Nos résultats sont regroupés dans le tableau III ci-après:

Tableau III. - Nombre de cas d'associations pour chaque espèce de parasite.

$\begin{array}{lll}\text { Type d'association } & \begin{array}{l}\text { Nombre de cas } \\ \text { avec association }\end{array} & \begin{array}{l}\text { Nombre de cas } \\ \text { sans association }\end{array}\end{array}$
A $\cdots \cdots \cdots \cdots \begin{cases}1 & \mathrm{AB} \\ 1 & \mathrm{AC} \\ 4 & \mathrm{AD}\end{cases}$
$1 \mathrm{AE}$
B $\ldots \ldots \ldots \ldots \ldots 1 \mathrm{AB}$
630
$\mathrm{C} \ldots \ldots \ldots \ldots 1 \mathrm{AC}$
$1 \quad 44$
$\mathrm{D} \ldots \ldots \ldots, 4 \mathrm{AD}$
1
$\mathrm{E} \ldots \ldots \ldots \ldots, 1 \mathrm{AE}$
$4 \quad 6$
$\begin{array}{ll}1 & 7\end{array}$

D'après ces résultats, nous constatons que les cas où l'on trouve 2 parasites d'espèces différentes dans un même hôte sont rares mais cependant possible. Des cas où l'on trouve plus de deux espèces à la fois n'ont pas été rencontrés.

\section{Influence des parasites sur l'état sexuel de l'hôte}

Nous n'avons pu envisager une étude de ce problème que dans le cas de Microphallus similis, étant donné la prédominance relative de ce parasite dans nos récoltes.

Influence sur le sexe mâle.

Il est nécessaire de séparer ici très nettement les mâles qui hébergent des sporocystes mûrs, de ceux très nombreux qui ne présentent que des sporocystes sans cercaires. 
Dans le premier cas on constate une castration parasitaire totale avec disparition totale, au moins au niveau des observations à la loupe binoculaire du testicule et de la vésicule séminale. Le pénis dans 249 des cas observés sur 277 au total n'était représenté que par la petite ébauche triangulaire qui caractérise chez les individus sains les mâles immatures. Un seul présentant à la fois testicule, vésicule séminale et pénis développés. 28 individus avaient des organes génitaux très regressés.

Pour les mâles présentant seulement des sporocystes sans cercaires mûres, sur 77 cas étudiés, un seul présentait l'état de castration largement caractéristique de la présence de cercaires mûres. Il est donc apparent que la castration parasitaire dépend de l'état d'évolution du parasite.

\section{Influence sur le sexe femelle.}

Dans tous les cas observés (259) l'ovaire disparaît pratiquement totalement et l'on ne trouve qu'exceptionnellement quelques embryons dans la cavité incubatrice (12 individus). Les cas représente manifestement un parasitisme relativement récent.

L'état des glandes annexes de l'appareil femelle est plus variable mais dans la majorité des cas elles se trouvent réduites à une petite structure transparente manifestement non fonctionnelle. Dans 47 cas seulement, elles apparaissent blanchâtres, comme chez l'individu sain, mais sont de taille très réduite.

\section{Étude des relations entre la taille de l'hôte et la présence des parasites}

Les individus recueillis (sains + parasités) ont une taille s'étageant entre $5 \mathrm{~mm}$ et $15 \mathrm{~mm}$ de hauteur de la coquille. Il a donc été possible dans les deux sexes de cal-

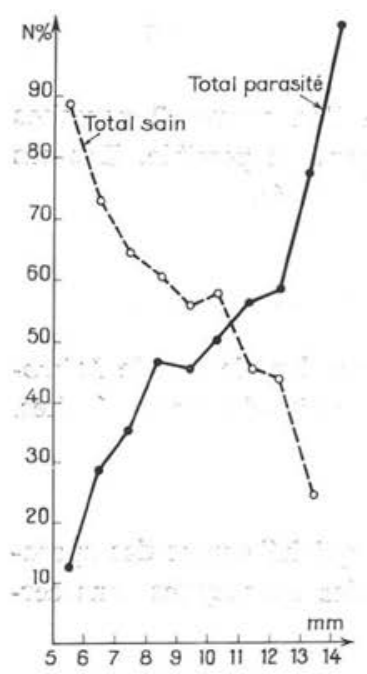
culer le taux d'infestation global des individus en fonction de leur taille (tableau IV et courbe).

Le tableau IV montre qu'il existe une augmentation régulière du taux de parasitisme avec la taille dans les deux sexes. Ces taux étant comme nous l'avons déjà vu pratiquement très voisins entre les deux sexes bien que légèrement plus élevés pour les femelles.

Cette observation est compatible avec l'hypothèse d'une susceptibilité à peu près constante au cours de la vie, un animal ayant d'autant plus de chances de se trouver parasité qu'il est plus âgé et le restant toute sa vie à partir de sa première infestation.

Courbe 1. - Fréquence des individus sains et parasités en fonction de la taille. 


\begin{tabular}{|c|c|c|c|c|c|c|c|c|c|c|c|c|}
\hline 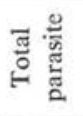 & & $\begin{array}{l}20 \\
\simeq \\
\simeq\end{array}$ & $\begin{array}{l}\stackrel{8}{0} \\
\hat{\infty} \\
\hat{i}\end{array}$ & $\frac{30}{ \pm 0}$ & $\begin{array}{l}0 \\
0 \\
0 \\
8 \\
y\end{array}$ & $\begin{array}{l}82 \\
\dot{y}\end{array}$ & $\begin{array}{l}20 \\
2 \\
g\end{array}$ & \begin{tabular}{l}
$\approx 0$ \\
\multirow{n}{n}{}
\end{tabular} & $\frac{3}{5}$ & $\begin{array}{l}0 \\
0 \\
0 \\
0\end{array}$ & 8 & \\
\hline 梠市 & & $\alpha$ & $\hat{n}$ & $\cong$ & $\underset{m}{\mathbb{N}}$ & $\begin{array}{l}\stackrel{8}{\sigma} \\
\qquad\end{array}$ & 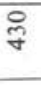 & $\stackrel{\infty}{\varnothing}$ & $\stackrel{\circ}{\circ}$ & $\tilde{\sim}$ & $n$ & $\begin{array}{l}8 \\
-\end{array}$ \\
\hline 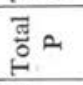 & & - & $\cong$ & $\overline{6}$ & $\bar{\Xi}$ & ర్లి & $\frac{N}{N}$ & 巳ั & N & 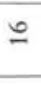 & in & $\stackrel{2}{2}$ \\
\hline 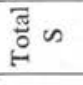 & & - & $\stackrel{\infty}{\sim}$ & $\stackrel{\infty}{=}$ & $\bar{ন}$ & $\stackrel{\infty}{\sim}$ & $\frac{\infty}{N}$ & $\mathscr{\infty}$ & ন & n & 0 & $\bar{\delta}$ \\
\hline$\stackrel{x}{\stackrel{\Xi \Xi ّ}{\Xi}}$ & . & & & & & & 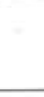 & & & & & \\
\hline 0 & & $m$ & ฉે & 5 & 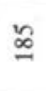 & สี & $\tilde{\sim}$ & \&̊ & $\bar{\lambda}$ & $\infty$ & $N$ & $\stackrel{\infty}{\infty}$ \\
\hline$a$ & & $\stackrel{2}{0}$ & $\frac{\therefore}{m}$ & $\begin{array}{l}30 \\
\infty \\
\text { N }\end{array}$ & $\frac{2}{n}$ & $\begin{array}{l}\text { ¿ी } \\
\text { ले }\end{array}$ & $\begin{array}{l}28 \\
8\end{array}$ & है & $\frac{2}{2}$ & $\begin{array}{l}\approx \\
\approx\end{array}$ & $\begin{array}{l}0 \\
8\end{array}$ & 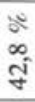 \\
\hline ro & & 0 & $a$ & તે & อิ & $\infty$ & $\Xi$ & $\stackrel{\infty}{\stackrel{\sim}{*}}$ & $\cong$ & 6 & $N$ & $\underset{\sim}{ \pm}$ \\
\hline$n$ & 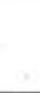 & & & & & & & & & & & \\
\hline$r_{0}$ & & $m$ & సి & $\ddot{\sigma}$ & $\stackrel{\varrho}{\varrho}$ & $\cong$ & $\stackrel{\infty}{=}$ & F & 0 & $\mathrm{~N}$ & 0 & $\frac{N}{n}$ \\
\hline 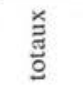 & & & & & & $=$ & & & & & & \\
\hline or & 0 & $n$ & त & ઝే & $\infty$ & ন্ & $\stackrel{2}{\cong}$ & ஃ & $\approx$ & $=$ & $m$ & $\underset{\infty}{\mathbb{0}}$ \\
\hline A & & 우 & $\begin{array}{l}30 \\
21\end{array}$ & $\begin{array}{l}\stackrel{2}{2} \\
2\end{array}$ & $\begin{array}{l}\dot{2} \\
\text { Z }\end{array}$ & $\begin{array}{l}\therefore 0 \\
\therefore\end{array}$ & $\begin{array}{l}\stackrel{\circ}{\sigma} \\
\stackrel{\sigma}{0}\end{array}$ & $\begin{array}{l}\text { OP } \\
\infty \\
i n\end{array}$ & $\begin{array}{l}80 \\
9\end{array}$ & $\stackrel{20}{E}$ & $\begin{array}{l}\text { ஃ̊ } \\
\&\end{array}$ & 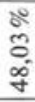 \\
\hline or & 0 & - & 0 & लै & $\infty$ & $\stackrel{m}{=}$ & $\approx$ & $\stackrel{0}{n}$ & $=$ & 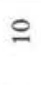 & en & $\frac{n}{\sigma}$ \\
\hline is & & & & & & & & & & & & \\
\hline or & 0 & $\nabla$ & $\cong$ & 요 & $\stackrel{\varrho}{\varrho}$ & $\Xi$ & 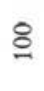 & \& & $\stackrel{\infty}{\sim}$ & $m$ & 0 & $\frac{g}{4}$ \\
\hline & $\begin{array}{l}\vdots \\
\vdots \\
\vdots \\
\stackrel{2}{!} \\
\stackrel{2}{\square}\end{array}$ & $\begin{array}{c}\vdots \\
\vdots \\
\frac{1}{n} \\
\frac{1}{n}\end{array}$ & $\frac{\vdots}{5}$ & $\begin{array}{c}\vdots \\
\vdots \\
\vdots \\
\infty \\
\stackrel{1}{n}\end{array}$ & $\begin{array}{l}\vdots \\
\vdots \\
\frac{9}{\infty} \\
\frac{9}{\infty}\end{array}$ & $\begin{array}{c}\vdots \\
\vdots \\
\frac{1}{2} \\
\frac{1}{2}\end{array}$ & 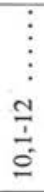 & $\begin{array}{l}\vdots \\
\vdots \\
\stackrel{5}{ \pm} \\
=\end{array}$ & $\begin{array}{c}\vdots \\
\vdots \\
\frac{m}{2} \\
\stackrel{m}{ \pm}\end{array}$ & $\begin{array}{l}\vdots \\
\vdots \\
\frac{1}{2} \\
\frac{\square}{2}\end{array}$ & $\begin{array}{c}\vdots \\
\vdots \\
\frac{2}{ \pm} \\
\frac{2}{ \pm}\end{array}$ & $\begin{array}{l}\vdots \\
\vdots \\
\vdots \\
\text { छू }\end{array}$ \\
\hline
\end{tabular}




\section{Discussion}

L'étude des formes larvaires hébergées par le Mollusque Gastéropode Littorina saxatilis (Olivi, 1792) de la région de Roscoff (Finistère), faite d'octobre 1971 à septembre 1972, nous a amenés aux conclusions suivantes:

En ce qui concerne les parasites eux-mêmes : 11 espèces ont été dénombrées. Leur taux de présence variait de $0,25 \%$ à $79,86 \%$. Il était de 79,86\% pour Microphallus similis Jaegers-Kioeld, 1900 et de 6,6\% pour Renicola roscovita : Stunkard, 1932. Ces espèces sont en fait les plus représentées dans la population étudiée. Notons que les valeurs observées chez Microphallus similis sont assez comparables à celles données par James (1964a-1968d), chez Littorina saxatilis sur la côte anglaise à Cardigan Bay, Whales.

Les associations entre cercaires sont rares sauf peut-être pour Renicola roscovita et Microphallus similis pour lesquels nous avons compté 4 cas d'associations sur 6 ou Renicola roscovita était présenté. Mais le faible nombre d'échantillons de ce type ne nous permet pas d'affirmer qu'il existe une association orientée, nous pouvons conclure que la double infestation est donc possible. James (1969) trouve aussi des cas de double ou même de triple infestations.

Les associations de cercaires avec des métacercaires sont rares sauf pour Renicola roscovita peur lequel nous avons compté 6 cas avec métacercaires et 4 seulement sans métacercaire.

Nous n'avons, pour notre part, pas noté de variations dans les pourcentages de parasitisme des prélèvements effectués tout au long de l'année. Nous pensons que la population étudiée, conserve un taux de parasitisme à peu près constant.

Nos résultats diffèrent de ceux de James (1969), qui trouve une variation saisonnière inverse de celle de la variation du cycle sexuel. Il relie ceci au fait que seuls les individus qui ont été accouplés sont susceptibles d'être infestés.

En ce qui concerne l'action du parasite sur l'hôte, quel que soit le type de parasite considéré, il y a toujours un effet de castration très prononcé du parasite sur le mollusque.

La relation «taille parasite » n'a pas été établie; nous ne pouvons ici qu'émettre une hypothèse : des élevages de 6 mois en aquarium nous ont montré que des animaux parasités n'étaient pas déparasités à l'issue de cette période. Nous pouvons supposer que, s'il n'existe pas de possibilité de se déparasiter pour un animal infesté, le parasitisme a d'autant plus de chance de s'exprimer chez l'animal âgé de grande taille que chez l'animal jeune.

La moyenne de taille des hôtes hébergeant Microphallus similis est de 9,75 mm, celle des hôtes hébergeant Renicola roscovita est de 11,09 mm.

James (1969) dans ces résultats ne trouve aucun parasitisme chez les formes juvéniles et $100 \%$ chez les individus les plus âgés. Il suppose que les jeunes sont résistants .et que les adultes sont de plus en plus sensibles à l'infestation. D'autre part, les adultes 
auraient plus de chance d'être infestés parce qu'ils sont exposés depuis plus longtemps au parasitisme. Cette dernière hypothèse rejoint la nôtre. Rappelons qu'il existe par ailleurs d'autres cercaires: Parvatrema homoetecnum James et Microphallus pygmeus (Levinsen) (James $1968 a, b)$ qui parasitent au contraire Littorina saxatilis dans leur forme juvénile, les adultes étant de plus en plus résistants en vieillisant.

Rothschild (1936-1941) qui a étudié ce phénomène chez Littorina neritoïdes et Hydrobia ulvae, propose d'admettre un accroissement du métabolisme chez les animaux parasités, ce qui entraìnerait une augmentation de la taille. Cette hypothèse ne paraît pas vérifiée par les travaux récents. L: métabolisme des animaux sains semblerait peu différent de celui des animaux parasités.

Fischer-Piette, Gaillard et James, 1964, ont trouvé que la longueur des radula des spécimens de Littorina saxatilis parasités était inférieure à celle des spécimens sains. Ils suggèrent que les larves de digènes causent la diminution de taille de la radula ou plus probablement entraîne l'augmentation de taille des coquilles des mollusques parasités. Ceci confirmerait l'hypothèse de Rotschild.

Nous n'avons pas noté l'influence du sexe sur le parasitisme dont les taux restent sensiblement identiques chez le $\sigma^{\circ}$ et la $\$\left(384 \delta^{*}\right.$ et $\left.377 \%\right)$.

Nous avons noté des taux élevés de sporocystes immatures chez les mâles. Ceci permettrait peut-être de conclure à la croissance beaucoup plus rapide du parasite chez les femelles que chez les mâles.

\section{Conclusion}

Nous avons étudié dans la région de Roscoff quelques trématodes parasites de Littorina saxatilis et leurs effets sur cet hôte.

- 11 espèces de cercaires ont été mises en évidence.

- La double infestation de type cercaire-cercaire ou cercaire-métacercaire a été rarement rencontrée.

- Microphallus similis était présente à un taux de parasitisme nettement plus .élevé que celui des autres cercaires. Il atteignait 79,86\% dans les stations choisies. Nos résultats peuvent donc pratiquement totalement lui être attribués.

- Nous avons aussi noté qu'il existe une certaine stabilité du taux de parasitisme tout au long d'une année dans une même population.

- Par ailleurs, un effet de castration important sur l'hôte dû à la présence de parasites a été observé.

- Nous avons aussi remarqué que le taux de parasitisme était plus élevé chez les formes âgées que chez les formes jeunes mais nous n'avons pas noté de différence entre les taux de parasitisme mâle et femelle.

Il y aurait par ailleurs une croissance beaucoup plus rapide du parasite chez les femelles que chez les mâles. 


\section{Bibliographie}

1. Bergerard (J.), 1971. - Facteurs écologiques et cycles sexuels de Littorina saxatilis, Olivi. Cah. Biol. Mar., 12, 187.

2. Bergerard (J.), 1975. - Cycle sexuel saisonnier dans une population naturelle de Littorina saxatilis (Olivi) (Gastéropode, prosobranche). Bull. Soc. Zool. Fr., 100, 133.

3. Fischer-Piette (E.), Gaillard (J.) et James (B. L.), 1964. - Etudes sur les variations. de Littorina saxatilis VI. Quelques cas qui posent de difficiles problèmes. Cah. Biol. Nat., 5, 125-171.

4. James (B. L.), $1964 b$. - Studies on larval trematodes from some littoral molluscs. Ph. D. Thesis. University College of Whales, Aberystwyth. $1968 a$. - The occurence of Parvatrema homoeotecnum James, 1964 (Trematoda: Gymnophallidae). In a population of Littorina saxatilis tenekosa (Mout.). J. Nat. Hist. 2, 21-37. 1968 b. - Studies on the life on Microphallus Pigmeus (Levinsen, 1888) (Trematoda: Microphallidae). J. Nat. Hist. 2, 155-172. 1968 d. -- The occurence of Larval Digenea in ten species of intertidal prosobranch molluscs in Cardigan Bay. J. Nat. Hist. 2, 329 . 343. 1969. - The Digenea of the Intertidal Prosobranch, Littorina saxatilis (Olivi) Sanderdruck aus Z. F. Zool. Systematik. Evolutions forschung Bd., 7, H. 4, S, 273316.

5. Lebour (M. V.), 1907. - Larval trematodes of the Northumberland coast. Trans. Nat. Hist. Soc. Northumberl., N. Set., 1, 437-454, 500-501.

6. RothsChiLD, 1941. - The effect of trematode parasites on the Littorina neritoildes (L.). S. Mar. Biol. Ass. U.K., 25, 69-80.

7. Stunkard (H. W.), 1932. - Some larval trematodes from the coast in the region of Roscoff, Finistère, Parasitology, 24, 321-343.

8. Willey (C. H.), Gross (P. R.), 1957. - Pigmentation in the foot of littorina littorea as. a means of recognition of infection with trematode larvae. J. Parasit., 43, 324-327. 